This is a postprint of an article published in

Niesner, R., Gericke, K.-H.

Fluorescence lifetime imaging in biosciences: Technologies and applications (2007) Frontiers of Physics in China, 3 (1), pp. 88-104. 


\title{
Fluorescence lifetime imaging in biosciences: technologies and applications
}

\author{
Raluca Niesner $^{1}$ and Karl-Heinz Gericke ${ }^{2 *}$ \\ ${ }^{1}$ Helmholtz Centre for Infection Research, Inhoffenstraße 7, D-38124 Braunschweig, Germany \\ ${ }^{2}$ Institute of Physical and Theoretical Chemistry, University of Braunschweig, Hans-Sommer-Strasse 10, D- \\ 38106 Braunschweig, Germany
}

\section{Short title:}

Fluorescence lifetime microscopy in biosciences

\section{Correspondence address:}

Karl-Heinz Gericke, Prof. Dr.

Technical University Braunschweig

Institute for Physical and Theoretical Chemistry

Hans-Sommer-Strasse 10

D-38106 Braunschweig

Germany

phone: +49 (531) 391 5326/25

fax: $\quad+49$ (531) 3915396

e-mail: k.gericke@tu-braunschweig.de 


\begin{abstract}
The biosciences require the development of methods, which allow a non-invasive and rapid investigation of biological systems. In this frame high-end imaging techniques allow an intravital microscopy in real-time, providing information on a molecular basis. Far-field fluorescence imaging techniques are some of the most adequate methods for such investigations. However, there are great differences between the common fluorescence imaging techniques, i.e. wide-field, confocal one-photon and two-photon microscopy, respectively, as far as their applicability in diverse bioscientific research areas is concerned. In the first part of this work, we briefly compare these techniques. Standard methods used in the biosciences, i.e. steady-state techniques based on the analysis of the total fluorescence signal originating from the sample, can successfully be employed in the study of cell, tissue and organ morphology as well as in monitoring the macroscopic tissue function. However, they are mostly inadequate for the quantitative investigation of the cellular function at molecular level. The intrinsic disadvantages of steady-state techniques are counteracted by using time-resolved techniques. Among these the fluorescence lifetime imaging (FLIM) is currently the most common. Different FLIM principles as well as applications of particular relevance for the biosciences, especially for fast intravital studies are discussed in this work.
\end{abstract}

Keywords: time-resolved fluorescence microscopy, fluorescence lifetime imaging, multi-focal twophoton microscopy, time-gating 


\section{Introduction}

A central methodological aim of biosciences is to experimentally simulate, as good as possible, the real environmental conditions of biological systems in order to gain a true image of the effects and phenomena of interest on both macroscopic and molecular level. Thus, the bioscientific and biomedical research is moving from ex vivo observations towards intravital investigations, i.e. investigations in the living organism. In this frame, the development of imaging techniques, which allow an accurate and highly sensitive monitoring of changes in the studied system without disturbing the natural processes, is of particular relevance. Fluorescence far-field imaging techniques like wide-field microscopy ${ }^{1}$, confocal one-photon microscopy ${ }^{2,3}$ and multi-photon laserscanning microscopy ${ }^{4}$ belong to the most adequate and most used imaging techniques in the biosciences due to their high potential for mapping biological systems in a non-invasive way and with high (spatial and temporal) resolution. However, there are significant differences as far as their optical performance, i.e. spatial resolution, imaging depth, signal-to-noise ratio, detection and recording efficiency as well as sensitivity and imaging speed are concerned. We briefly review the advantages and restrictions of commonly employed fluorescence far-field microscopic techniques for diverse biological application areas. Special attention is dedicated to the comparison of two different setups of the two-photon laser-scanning microscopy, i.e. single-beam scanning combined with photomultiplier detection and multi-beam scanning combined with CCD detection, as far as imaging depth and imaging speed are concerned.

Although steady-state techniques, i.e. techniques which use only the integral emission and the spectral information contained therein, are well established investigation tools for biosciences, they show intrinsic limitations in quantitatively monitoring the cellular function on molecular basis, for instance in the investigation of intracellular fluorescence resonant energy transfer (FRET) ${ }^{5}$. The reasons therefore are twofold:

- in many applications the absorption and emission spectra of different chromophores within a cell overlap so that the chromophores can hardly be resolved and

- modifications of the fluorescence intensity are caused not only by changes in the chromophore concentration but also by photo processes like quenching, internal conversion or other types of intermolecular energy transfer, which cannot be resolved without previous calibration.

The interest in time-resolved measurements mainly originates from the information available in the fluorescence decay of the sample. This is molecule-specific and highly sensitive to modifications of the microenvironment as well as to changes induced by photo processes, e.g. quenching or FRET, 
but it is hardly affected by fluctuations of the chromophore concentration, which implies highaccuracy calibration-free measurements. Thus, fluorescence lifetime imaging (FLIM) can in many cases counteract the disadvantages of steady-state techniques ${ }^{6,7}$ and, consequently, has become a versatile tool in the quantitative measurement of cellular parameters as well as in monitoring and quantifying molecular phenomena within the cell.

The focus of this work lies on the most used FLIM techniques, i.e. homodyne and heterodyne frequency-domain FLIM ${ }^{8}$, time-correlated single-photon counting (TCSPC) ${ }^{9,10}$ and time-gated techniques ${ }^{11,12}$ as well as on their compatibility with current high-end far-field imaging techniques with particular relevance for the biosciences. The comparison criteria refer to standard fluorescence imaging parameters, i.e. speed and optical performance, and also to specific features for timeresolved measurements, e.g. processing and evaluation speed and accuracy as well as temporal resolution. Furthermore, since biological systems contain numerous endogenous cromophores apart of the fluorescing markers, the fluorescence decay in such samples is usually multiexponential. Thus, it is important for the FLIM techniques to be able to determine the form of the intensity decay law and to interpret the decay in terms of molecular features of the sample. Two concrete applications of time-gated FLIM based on ICCD detection combined with multi-focal two-photon laser scanning microscopy, i.e. a novel technology with great potential for intravital time-resolved investigations in real-time, are also presented. 


\section{Fluorescence imaging techniques}

Since the questions about the structure and (molecular or macroscopic) function of biological systems are manifold, the requests on the fluorescence microscopy techniques are very different depending on the sample or phenomenon under study. Standard low-cost microscopy methods used in the biosciences are wide-field techniques based on one-photon excitation ${ }^{1,13-15}$, which do not specially need lasers as excitation sources but can also use flash lamps, LEDs or mercury lamps. The typical detectors in wide-field imaging experiments are cameras. These techniques allow only a coarse representation of the investigated sample because they are characterised by a rather poor lateral resolution and practically no axial resolution. Moreover, using these methods based on $\mathrm{UV} /$ visible excitation, the deeper layers of the sample cannot be imaged because the excitation light is completely scattered already in the upper layers, i.e. imaging tissue or intact organs is impossible ${ }^{4,16}$. Although the use of lamps or LEDs instead of lasers insures low photobleaching of the samples ${ }^{1}$, it also limits the sensitivity of the device, i.e. faintly fluorescing samples cannot be visualised.

The confocal microscopy based on one-photon excitation, which is a scanning technique, provides a very good (diffraction limited) lateral resolution, an adjustable axial resolution, which depends on the dimension of the pinhole in front of the detector, and due to the use of UV/visible laser radiation for excitation, it is appropriate for imaging low fluorescence signals in isolated cells or, generally, in thin samples ${ }^{2,3,17-19}$. However, this is also a UV/visible technique and, thus, it does not allow the visualisation of intact tissue or organs. The use of laser radiation combined with a large excitation volume along the optical axis leads to dramatic photodamage and photobleaching effects within the sample ${ }^{4,16}$. Furthermore, since only point detectors, i.e. photomultiplier tubes (PMT) or photodiodes, can reasonably be employed for the confocal alignment, this method uses point-bypoint scanning and detection and, thus, it is very slow ${ }^{4,16}$. The scanning in confocal microscopy is realised either by moving the sample itself (very slow but optically stabile) or by moving the excitation laser beam over the resting sample (instrumentally more complex but faster) ${ }^{2,17}$. Line scanning based on cylindrical optics is a fast alternative currently used in some commercial microscopes (LSM live, Zeiss, Jena, Germany). However, the resolution along the scanning line is rather low.

Since the early nineties, the two-photon (multi-photon) laser-scanning microscopy (TPLSM) has been established as a versatile imaging technique for biological systems with a high potential not only for organ but also for intravital (i.e. in the living organism) multi-dimensional imaging, although its lateral resolution is slightly lower than that achieved in confocal microscopes due to the longer excitation wavelength ${ }^{4,16}$. 
The advantages of TPLSM over standard one-photon excitation fluorescence microscopic techniques, i.e. confocal and wide-field microscopy, are the intrinsic 3D spatial resolution, a large penetration depth in thick highly-scattering biological media and a low photobleaching and photodamage of the sample outside the focal plane. These advantages are to be derived from the principal characteristics of the two-photon excitation. The low two-photon excitation cross-section and the quadratic dependence of the excitation rate on the illumination photon flux lead to a 3Dconfined excitation volume and, thus, to almost no photoprocesses outside the focal plane. Since biological tissue can be easier penetrated by near infrared (NIR) than by visible or ultraviolet light, the necessity of using NIR illumination for the two-photon excitation lead to a large penetration depth in this kind of samples. However, low two-photon excitation cross-sections also lead to the main drawback of TPLSM, i.e. the need of high photon fluxes for adequate excitation rates. This drawback is counteracted by the use of ultra-short (femtosecond) pulsed lasers, characterised by a low averaged energy in spite of high photon fluxes during the laser pulse. Considering the advantages of the two-photon excitation over the one-photon excitation, one would expect that three- or more-photon excitation is even more adequate for imaging biological samples. However, the excitation cross-section in these cases is very low, so that the photon fluxes necessary for sufficient excitation rates can hardly be achieved ${ }^{4,16}$.

Currently, steady-state TPLSM techniques based on scanning of the sample with a single laser beam followed by fluorescence detection by means of photomultiplier tubes (PMT), i.e. point detection, are largely employed in biosciences. However, due to the fact that the usually employed galvanometer scanners in combination with point-by-point detection are too slow, these techniques cannot track the dynamics of important biological processes. Devices, which allow a faster scanning of the sample, e.g. acusto-optical deflectors, are good alternatives to the standard galvanometer scanners, but apart of their complicated and sometimes unstable instrumental implementation, they still have the disadvantage that due to photodamage only $10 \%$ of the full laser power can be used for biological investigations ${ }^{20,21}$. Techniques based on a multi-beam scanning combined with synchronous fluorescence detection, i.e. based on CCD cameras, are able to use the full laser power under non-invasive conditions for the biological samples while being very fast and, thus, represent very advantageous TPLSM techniques for biosciences. The innovative aspect of the multifocal technique (Fig. 1) employed in our experiments is that it avoids cross-talk, i.e. interference between neighbouring beam lets, due to multiplexing ${ }^{21}$.

Insert Fig. 1 
The special part of the multifocal system used by us is the beam-multiplexer that is able to split up an incoming NIR beam (710-1050 nm) into two beam sets each consisting of up to 32 beams. Thereby one set features s-polarisation while the other one is p-polarised. Both sets are recombined and coupled into an upright microscope through the scan lens - tube lens combination (SL, TL). A dichroic mirror (DM) reflects the excitation light towards the objective lens that focuses it onto the sample. The geometry of the beam-multiplexer generates a single line of up to 64 foci in the object plane whereby adjacent foci have opposite polarisation and a typical distance of $1.5 \mu \mathrm{m}$ from each other for a $20 \times$ objective lens (NA = 0.95). In addition, the degree of parallelization can be changed from 64 beams down to 32, 16, 8, 4 and also to a single one whereas the power per beam is doubled with each time the number is halved. Thereby the length of the line is reduced while the spacing between adjacent foci remains constant. Scanning is done with a conventional pair of galvanometer scanners, which insure a high stability of the image in contrast to acusto-optical deflectors. In combination with the motorized microscope z-drive three-dimensional objects can be visualized.

In the presented microscope (Fig. 1), the fluorescence is collected by the objective lens and can be imaged onto one or two CCD cameras. A switchable dichroic mirror splits it up onto the two cameras enabling simultaneous acquisition of two different colors. Each camera has a filter wheel in front of it to further separate the emission light. In addition, another emission pathway changer allows switching from CCD detection mode to non-descanned PMT detection.

We already compared in detail a standard single-beam PMT with a multiplexing multi-beam CCD technique (MB-CCD), as far as technical characteristics important for the bioscientific research are concerned, i.e. spatial resolution, penetration depth, signal-to-noise ratio (SNR) and imaging speed ${ }^{22}$. While the spatial resolution is the same no matter of detection or excitation pattern, the singlebeam scanning combined with PMT detection turned out to be more appropriate for deep-imaging experiments (more than $200 \mu \mathrm{m}$ depth) because in this imaging depth the SNR of the single-beam PMT setup is clearly better than that of the MB-CCD setup. As far as the imaging speed is concerned, it is undisputable that the MB-CCD technique alone allows real time measurements in fast biological systems, e.g. flow within a blood vessel (Fig. 2).

Insert Fig. 2

Two-photon techniques, which break the diffraction resolution limit, are based on point spread function engineering and must be mentioned when giving a general review of imaging techniques for biosciences. $4 \pi$ microscopy, STED microscopy and triple-state depletion microscopy are just 
some of these methods, which have been especially developed in the group of Stefan Hell (Max Plank Institute, Goettingen, Germany) ${ }^{23-25}$. These techniques are particularly appropriate for optical imaging on the nanometer scale but are completely inadequate for measurements in (usually) thick biological samples.

\section{Principle of Fluorescence Lifetime Imaging (FLIM)}

The molecular parameter of interest in this work is the fluorescence lifetime $\tau$ of endogenous or exogenous chromophores within cells, which strongly depends on the cellular environment, i.e. proximity to loaded macromolecules like proteins, $\mathrm{pH}$, viscosity, ion concentration, etc. The definition of the fluorescence lifetime is ${ }^{26}$ :

$$
\tau=\frac{\phi}{k_{F}} \text { with the fluorescence quantum yield } \phi=\frac{k_{F}}{k_{F}+k_{I S C}+k_{S E}+k_{\text {bleaching }}+k_{\text {chem }}+k_{n r}} .
$$

A simplified Jablonski diagram (Fig. 3) best explains this mathematical formula and illustrates the pathways through which the environment influences the fluorescence lifetime.

\section{Insert Fig. 3}

The simplified fluorescence decay of a fluorophore in a homogenous media is given by:

$$
F(t)=I_{0} \cdot e^{-t / \tau} \cdot e^{-t / \theta} .
$$

$\theta$ is the rotational correlation time of the molecule and the exponential term containing it describes the rotational diffusion. The main simplification in this formula is that it neglects the fact that the fluorescence anisotropy has a multiexponential behaviour depending on the symmetry of the chromophore and on the excitation mode.

When the emitted light is detected under the magic angle $\left(54.7^{\circ}\right)$, at which polarisation effects are minimised, i.e. effects of the rotational diffusion caused by different viscosities of the media or by binding to macromolecules or to membranes, the fluorescence decay is simplified to ${ }^{26}$ :

$$
F(t)=I_{0} \cdot e^{-t / \tau} \text {. }
$$

Even if experiments are not performed under the magic angle, the effect of rotational diffusion is negligible when this is much faster or much slower than the fluorescence decay ${ }^{26}$. 
Biological systems resemble multiexponential fluorescence decays since they contain a complex mixture of endogenous chromophores, e.g. NADH, NADPH, FAD, serotonin, melanin, tryptophan and tyrosine in proteins or porphyrins ${ }^{27}$ :

$$
F(t)=\sum_{i} a_{i} \cdot e^{-t / \tau_{i}}
$$

$\tau_{i}$ is the fluorescence lifetime of the chromophore $i$ and $a_{i}$ is the corresponding weighting factor.

Since both the endogenous and the exogenous chromophores are usually heterogeneously distributed within the cell, tissue or organ, bulk measurements of the fluorescence lifetime cannot provide sufficient information about the real cellular phenomena. Only imaging experiments, i.e. fluorescence lifetime imaging - FLIM, allow a complete, bioscientifically relevant picture of the samples under study ${ }^{6,8,26,28}$.

There are two principal techniques to determine the fluorescence lifetime in an image: frequencydomain and time-domain FLIM techniques. Further techniques like those based on the stimulated emission depletion ${ }^{29}$ have their own attractiveness but are for most users less interesting since they are instrumentally complex and often unstable.

\subsection{Frequency-domain FLIM}

In frequency-domain (FD) FLIM techniques, the sample is excited with light, which intensity is modulated at a high frequency comparable to the reciprocal of the fluorescence lifetime $\tau$. The subsequent emission of the sample is also intensity-modulated at the same frequency. However, this emission does not precisely follow the excitation but rather shows phase delays and amplitude changes which are determined by the fluorescence decay law of the sample, i.e. the phase of the emission is shifted to later time points as compared to the excitation light, whereas the peak-to-peak height of the modulated emission is decreased relative to that of the modulated excitation ${ }^{6-8,26,28}$.

At each modulation frequency, the phase shift increases from 0 to $90^{\circ}$ with increasing modulation frequency $\omega$ and corresponds to the time-delay between excitation and emission. This finite time response of the sample also results in demodulation of the emission by a factor $m_{\omega}$. This factor decreases from $1(100 \%)$ to $0(0 \%)$ with increasing modulation frequency. At low frequencies, the emission immediately follows the excitation, i.e. a phase angle (phase shift) near to zero and a demodulation factor near to 1.0. As the modulation frequency is increased, the finite lifetime of the excited state prevents the emission from precisely following the excitation. Thus, for a single- 
exponential decay, the phase shift and the demodulation factor measured at a particular frequency are related to the fluorescence lifetime as follows ${ }^{6,7,28}$ :

$$
\begin{gathered}
\tan \phi_{\omega}=\omega \cdot \tau \\
m_{\omega}=1 / \sqrt{1+\omega^{2} \cdot \tau^{2}} .
\end{gathered}
$$

For multiexponential decays the lifetime calculated as described above are the result of a complex weighting of various components in the emission. For such samples it is necessary to measure the phase shift and demodulation values over the widest possible range of modulation frequencies with the centre frequency being comparable to the reciprocal of the mean decay time of the emission.

The FD data for an intensity decay can be calculated from the sine and cosine transforms of $F(t)$ $6,7,28$.

$$
N_{\omega}=\frac{\int_{0}^{\infty} I(t) \cdot \sin \omega t \cdot d t}{\int_{0}^{\infty} I(t) \cdot d t}, \quad D_{\omega}=\frac{\int_{0}^{\infty} I(t) \cdot \cos \omega t \cdot d t}{\int_{0}^{\infty} I(t) \cdot d t} .
$$

For a multiexponential decay these transforms are related to the fluorescence lifetimes $\tau_{I}$ and the weighting factors $a_{i}$ :

$$
N_{\omega}=\sum_{i} \frac{a_{i} \cdot \omega \cdot \tau_{i}^{2}}{\left(1+\omega^{2} \cdot \tau_{i}^{2}\right)} / \sum_{i} a_{i} \cdot \tau_{i} \quad D_{\omega}=\sum_{i} \frac{a_{i} \cdot \tau_{i}}{\left(1+\omega^{2} \cdot \tau_{i}^{2}\right)} / \sum_{i} a_{i} \cdot \tau_{i} .
$$

The calculated frequency-dependent values of the phase angle $\phi_{c \omega}$ and of the demodulation factor $m_{c \omega}$ are given by:

$$
\begin{aligned}
& \tan \phi_{c \omega}=N_{\omega} / D_{\omega} \\
& m_{c \omega}=\sqrt{N_{\omega}^{2}+D_{\omega}^{2}} .
\end{aligned}
$$

It is consequently obvious that resolution of a complex decay requires multiple measurements at a number of modulation frequencies, which span the frequency response of the sample.

First useful variable-frequency instruments were developed in the groups of Gratton and Lakowicz 6-8,26,28. They allowed phase and modulation measurements from 1 to $200 \mathrm{MHz}$. Currently, devices which work at frequencies up to $10 \mathrm{GHz}$ exist ${ }^{26}$. However, multiexponential evaluation is more difficult at high modulation frequencies when the phase angle approaches $90^{\circ}$, since the requirements on phase angle resolution for accurately determining the fluorescence lifetimes and the corresponding weighting factors increase over the technical possibilities ${ }^{8,26}$. 
As excitation sources for FD FLIM one can employ continuous wave lasers, pulsed lasers, e.g. cavity-dumped dye lasers or Ti:Sa laser ${ }^{6-8,28,30,31}$, as well as low-cost light-sources, e.g. flash lamps or even LEDs ${ }^{32}$. Thus, the possibility to build a low-cost FLIM device makes the FD techniques especially attractive for routine applications.

While the early frequency-domain devices based on Debye-Sears modulators allowed modulation of the excitation only at few fixed frequencies, current frequency synthesisers, i.e. instruments based on a phase shifter which add a small frequency to a high-frequency ( $\mathrm{MHz}$ ) device, can allow modulation at closely spaced frequencies over a large range ${ }^{8,26}$. Currently, the modulation of the excitation light is done either with acusto-optical ${ }^{30,33-37}$ or with electro-optical modulators ${ }^{6-8,26,28,32}$. While acusto-optical modulators provide modulation only at discrete resonances (the RF modulation frequency and its harmonics) in a limited range of frequencies, electro-optical modulators provide almost continuous modulation over a large range of frequencies. However, the latest necessitate high input voltages of 1 to $7 \mathrm{kV}$, which make their use somewhat difficult.

In order to detect the modulated emitted light and to obtain the phase and modulation information, the gain of the detector must be also (sinusoidal) modulated at the same frequency as the excitation light, i.e. homodyne FD techniques ${ }^{30,34}$, or at a frequency slightly different from that of the excitation, i.e. heterodyne FD techniques based on cross-correlation ${ }^{8,26}$. The use of crosscorrelation results in the rejection of harmonics and other sources of noise of the emitted light, so that there is no need for accurate sinusoidal modulation, i.e. even a laser pulse train can be used. The basic idea is to modulate the gain of the detector at a frequency offset ( $\delta F$ between 10 and 100 $\mathrm{Hz}$ ) from the excitation light modulation frequency. The result is a low-frequency signal from the PMT at frequency $\delta F$ that contains the phase and modulation information, i.e. fluorescence decay information. The phase and modulation of this low-frequency signal can be easily measured either by analog or digital methods. Currently, this signal is digitized and analyzed by a fast-Fourier transform ${ }^{8,26,38}$.

Standard detectors for FD FLIM techniques are different types of PMT although lately also modulated intensified CCD cameras (for both homodyne and heterodyne detection) have been used ${ }^{8,9,26}$. The main argument against intensified CCD as detectors for FD FLIM is their relatively low sensitivity ${ }^{9}$. The time-resolution, when using photomultiplier tubes, is determined by their transit time spread (TTS) of the detector. For dynodes PMT this is maximally $200 \mathrm{MHz}$ (5 ns), whereas for MCP PMTs it can easily reach $2 \mathrm{GHz}$ (500 ps) ${ }^{8,26}$. Although MCP PMT seems to be the ideal 
detector for FD FLIM, its use for the more advantageous heterodyne detection has the disadvantage that the MCP PMTs need special self-designed circuits for cross-correlation outside the detector ${ }^{26}$. Consequently, $200 \mathrm{MHz}$ (5 ns) is the limit of most commercial FD devices out of two reasons:

- it is difficult to obtain electro-optical modulators over this frequency due to their high halfwave voltages and

- most photomultiplier tubes have their upper frequency limit at $200 \mathrm{MHz}$.

Gigaherz modulation can be achieved by using the harmonic frequency content of a laser pulse train, e.g. from a mode-locked Nd:YAG pumping a cavity-dumped dye laser or from a Ti:Sa laser 8,30,34. In this case, the detection must be performed with MCP-PMT, which are characterised by a narrow TTS and which allow ps time-resolution.

In order to obtain the fluorescence lifetime information from FD FLIM data, one has to perform a comparison between the emission of the sample and the scattered light similarly to TCSPC, as it will be discussed later. Since the response function of the most PMT strongly depends on the detected wavelength, this comparison implies complicated measurements, which easily leads to instrumental instability ${ }^{6,8,26}$. Furthermore, the background in FD measurements cannot just be subtracted from the total signal like in time-domain measurements, since the first can contain different frequencies, e.g. of scattered light or of fluorescing cellular components, which make the measurements once more complicated ${ }^{39}$.

Since imaging techniques for biosciences should be able to track fast dynamics of biological phenomena, their data-acquisition speed is an important issue. FD FLIM techniques which employ PMT (or photodiodes) as detectors are intrinsically slow techniques compared to those techniques, which employ ICCD cameras. This speed advantage of these synchronous detectors (cameras) as compared to point-detectors is maintained despite the current developments of FD FLIM techniques, which allow multi-dimensional, i.e. in xyz space and frequency, data-acquisition based on multi-channel detection ${ }^{8,30,33-37}$. Furthermore, only ICCD cameras can be used as detectors in multi-focal microscopy, i.e. faster microscopy, so that fast detection can be combined with a fast scanning for imaging samples in real time. However, multi-focal FD FLIM has not been reported yet.

\subsection{Time-domain FLIM}

FLIM in time-domain is primarily more advantageous than FLIM in frequency-domain because it provides a direct measurement of the fluorescence decay and, thus, a direct access to the parameter/parameters of interest ${ }^{9,10,12,40-45}$. Considering the fact that many endogenous 
chromophores (quenched donors in FRET, NADH, NADPH) are characterised by fluorescence lifetimes in ps-range, the time-accuracy provided by FD FLIM is often insufficient. In contrast, TD FLIM techniques reach time-resolutions in the low ps-range or even fs-range ${ }^{8-11,40,45-47}$.

The standard TD FLIM technique, currently used in most bioscientific applications, is the time correlated single photon counting (TCSPC). However, time-gated techniques increasingly gain on relevance out of reasons which will be discussed in the following.

\subsubsection{Time-correlated single photon counting (TCSPC)}

Time-correlated single photon counting (TCSPC) is based on the excitation of the sample with a pulsed radiation source, the detection of single fluorescence photons, and the build-up of a histogram of the photon detection times. The result is the distribution of the photon density versus time, i.e. the fluorescence decay curve. Thereby, the molecules within the sample are repeatedly excited with the radiation of short pulsed high-repetition rate lasers in order to observe their fluorescence. Each laser pulse is optically monitored by a high-speed photodiode or photomultiplier to produce a start signal which is used to trigger the voltage ramp of a time-to-amplitude converter. The voltage ramp is stopped when the first fluorescence photon is detected. Finally an output pulse is provided whose voltage is proportional to the time between the start and the stop signals. A multichannel analyser converts this voltage to a time channel using an analog-to-digital converter. Summing over many pulses, the multichannel analyser builds up the fluorescence photon counting distribution. In order to avoid artefacts due to fluctuation of the pulse-amplitude, discriminators, e.g. leading-edge (LD) or constant fraction discriminators (CFD), are used. Currently, CFD are mostly met in TCSPC devices because their time-jitter (approx. 50 ps) is less influenced by the instrumental response function of the detector compared to LD (approx. $1 \mathrm{~ns}$ ) ${ }^{9,42,46}$.

Excitation sources for TCSPC are all types of high-repetition rate ps- and fs-pulsed lasers, e.g. cavity-dumped dye lasers, mode-locked cw lasers, pulsed tunable solid-state lasers like Ti:Sa lasers, fiber lasers or pulsed diode lasers, which makes TCSPC compatible both with the laser-scanning one-photon and two-photon excitation microscopy ${ }^{8,9}$. The detection in TCSPC is usually performed with photomultiplier tubes (PMT) but applications with photodiodes, especially avalanche photodiodes, have been reported ${ }^{8,9}$. However, the application of avalanche photodiodes is limited due to the poor time-resolution of these detectors, i.e. usually few $100 \mathrm{ps}^{9}$. Delay-line anode and quadrant anode PMT allowed the use of TCSPC in conjunction with wide-field imaging ${ }^{9}$. 
Traditional TCSPC techniques are especially appropriate to measure fluorescence lifetimes in bulk solutions of one or more chromophores. Since they allow the detection of only one photon every 100 pulses at high repetition rates like in standard TPLSM experiments, in which Ti:Sa lasers of approx. $80 \mathrm{MHz}$ repetition rate are employed, they are too slow to monitor even slow biological phenomena, f.e. the acquisition time of a 256x256 pixel image amounts to some ten minutes ${ }^{9,26}$.

Compared to traditional TCSPC setups the maximum photon count rate has been increased dramatically by using an innovative analog-to-digital conversion technique, which allows the detection of up to one photon every 10 pulses and, thus, make TCSPC compatible to imaging in biological systems ${ }^{9}$. For use in laser scanning microscopes the technique has been extended to the build-up of a three-dimensional photon density pattern containing the decay curve as well as the coordinates of the scanning area ${ }^{9,10,40,46,48}$.

This single-channel TCSPC imaging technique can be used with several detectors working in different wavelength intervals for spectrally-resolved multi-channel TCSPC or with identical detectors to increase the acquisition speed in standard multi-channel TCSPC. The technique works with both a multi-channel PMT and several individual PMTs and MCP-PMTs. It makes use of the fact that the detection of several photons in different detection channels in one laser period is unlikely. Therefore, the single photon pulses from all detector channels can be combined into a common photon pulse line and sent through the normal time measurement procedure of the TCSPC module ${ }^{10,40,46}$.

The output of each PMT channel is connected to a constant-fraction discriminator. When the PMT detects a photon, the corresponding discriminator triggers and sends a pulse to the subsequent encoding logic. The encoder delivers the number of the PMT channel that detected the photon. The channel number is used as an additional dimension in the multi-dimensional histogramming process of the TCSPC imaging technique. In the unlikely case that several detectors deliver output pulses in the same laser period, the enconder delivers a "don't count” signal that suppresses the recording of the event in the TCSPC module. The technique can reasonably be used for up to four individual ultrafast MCP-PMTs (e.g. R3809U by Hamamatsu) or one 16 channels or 32 channels multi-anode $\mathrm{PMT}^{10,40,46}$.

The recording electronics of the multi-detector TCSPC device consists of a time measurement channel, a scanning interface, a detector channel register, and a large histogram memory. The time measurement channel contains the usual TCSPC building blocks in the "reversed start-stop" 
configuration. For each photon, it determines the detection time $t$ with respect to the next laser pulse. The scanning interface is a system of counters, which receive the scan control signals (frame sync, line sync and pixel clock) from the optical scanners, usually galvanometer scanners. This determines the current location ( $x$ and $y$ ) of the laser focus in the scanning area. Synchronously with the detection of a photon, the detector channel number $n$ for the current photon is read into the detector channel register. If light is split into different wavelength intervals in front of the detectors, $n$ represents the wavelength of the detected photon ${ }^{10,40,46}$.

The obtained values for $t, x, y$ and $n$ are used to address the histogram memory, in which the distribution of the photons over time, detector (wavelength), and the image coordinates are stored. The result is a four dimensional data structure, which contains separate blocks for different wavelength intervals for spectrally-resolved multi-channel TCSPC. Each block can be regarded as an image containing a full fluorescence decay curve in each pixel ${ }^{10,40,46}$.

Most recent developments of the detection electronics allows count rates even higher than $10^{6}$ photons/s, i.e. processing of one photon per laser pulse at a repetition rate of $80 \mathrm{MHz}(2007$ LaVision Biotec, Bielefeld, Germany). An interesting perspective for increasing the dataacquisition speed is to combine multi-detector TCSPC with multifocal TPLSM. Apart of the speed gain and/or (spectral) information gain of the multi-channel TCSPC, this method is able to mitigate the disadvantage of pile-up of single-channel TCSPC, i.e. due to saturation effects at the detector, since the same fluorescence signal is divided on more detectors ${ }^{10,40}$.

The time-resolution of TCSPC is determined by the instrumental response function of the detector determined by the rising edge of the signal. While for dynode PMT the response function is usually asymmetrical and broad, MCP PMT are characterised by a narrow response function, which allows time-resolution of few 10 ps or even down to $800 \mathrm{fs}^{10,40}$.

In spite of the presented advantages of current TCSPC techniques for bioscientific investigations, there are still some aspects referring the TCSPC principle itself, which limits its applicability. In order to obtain feasible fluorescence decays, measurements of the sample and of a calibration reference, i.e. usually scattered excitation radiation, are necessary ${ }^{8,26,45}$. Since the IRF of most PMT strongly depends on the wavelength, this calibration is rather difficult especially in the two-photon microscopy because the PMTs are often not able to detect radiation at both the excitation and the emission wavelengths. Therefore, special short-living fluorescence probes are needed as calibration references. The necessity of a double measurement for determining the fluorescence decay 
intrinsically limits the speed of data-acquisition in TCSPC. Thus, current TCSPC techniques can be used to monitor relatively slow processes. They are especially adequate for applications, in which a high detection efficiency and sensitivity is requested, e.g. measurements of faintly fluorescing samples or in the single-molecule regime.

\subsubsection{Time-gated FLIM}

Time-gating techniques, i.e. single- and multi-gate imaging by means of either slow but highly

efficient point-detectors ${ }^{12,43,45}$ or by means of fast but low-efficiency image intensifiers ${ }^{11,41,49}$, are alternative TD FLIM techniques compatible with two-photon microscopy. Thereby, one or more time-gates are synchronised with the pulse train of a high-repetition rate pulsed laser, e.g. Ti:Sa laser. The fluorescence signal is registered only during these time-gates.

An important advantage of the time-gated techniques is that the shape of the instrumental response function of the detector does not lead to severe artefacts in the fluorescence decay. Thus, a deconvolution of the raw-data (required for FD FLIM and TCSPC) is not necessary. Consequently, spectrally induced artefacts in deconvolved fluorescence decays, which arise from a wavelength dependent response function, are not applicable ${ }^{12,45}$.

Typically less than $10 \%$ of the signal are registered in single-gate images. Thus, using pointdetectors is rather disadvantageous because their counting efficiency cannot be fully used. Thus, it is favourable to increase the imaging speed by using multi-gating techniques (up to 10 gates) ${ }^{45}$. However, even under these circumstances, the imaging speed of the point-detection time-gating techniques is rather low and inadequate for monitoring the molecular dynamics. Furthermore, multigating imaging implies the previous knowledge of the exponential behaviour of the sample, which obviously represents a major limitation in the study of biological systems ${ }^{9}$.

The time-resolution in these time-gated setups is mainly determined by the gate-width and not by the response function of the point-detector. Thus, there is an inverse proportionality between the time-resolution and the detection efficiency, since the last increases with increasing gate-width, whereas the first is higher at narrower gate-widths ${ }^{45}$.

Time-gated imaging based on intensified CCD cameras is characterized by a lower detection and counting efficiency compared to TCSPC or FD FLIM and by a poorer signal-to-noise ratio in deeptissue compared to techniques which employ point-detection. However, it is the best choice for 
investigating fast dynamics in moderated to brightly fluorescing biological samples. The principle of data-acquisition in time-gating FLIM based on ICCD detection is illustrated in Fig. 4.

Insert Fig. 4

The advantages of this method over other FLIM techniques ${ }^{11,49,50}$ are summarised as follows:

- The time-gated CCD technique is compatible and has been used in combination with multifocal two-photon laser scanning microscopy, so that high-speed imaging can be achieved (up to $2 \mathrm{~Hz}$ ).

- Multiexponential processing and evaluation can be easily performed as will be described in the following on the example of the redox NAD(P)H metabolism in MIN6 cells (mutated insulin producing pancreatic $\beta$-cells) by means of biexponential FLIM.

- The time resolution is limited by the time-jitter (less than $10 \mathrm{ps)}$ of the detector as was demonstrated in fluorescence lifetime measurements on a series of DASPI (4-[4(dimethylamino)styryl]-1-methylpyridinium iodide) solutions of different viscosities. Thereby, we made use of the fact that the internal torsion in the DASPI molecule decreases with increasing viscosity, which determines an increase of the fluorescence lifetime in the picosecond range.

- A spatial resolution as good as in steady-state multifocal two-photon microscopy can be achieved.

- There is no danger of artefacts of the fluorescence decay due to detector saturation or to pile-up effects.

\section{FLIM data evaluation}

Up to this point, we compared the common FLIM techniques as far as the accuracy and speed of data-acquisition and processing is concerned. However, a relevant comment on FLIM techniques must include a brief review of the evaluation procedures of the time-resolved (or frequencyresolved) fluorescence signal. This is particularly important since the data-evaluation may be either time-consuming and lead to a dramatic loss of speed in lifetime-image generation or numerically inaccurate. The evaluation of monoexponential decay curves is straightforward since it can be reduced to a linear regression analysis both in FD and in TD FLIM (provided the background can be neglected). Unfortunately, monoexponential decays are the exception rather than the rule in biosciences. 
The commonly used evaluation techniques of multiexponential decays in both FD and TD FLIM are iterative procedures based on the least-squares method. ${ }^{26,34,51,52}$ This method is accurate, but time consuming, sometimes unstable and the global minimum might be missed.

Global analysis techniques imply the measurement of the same sample at different wavelengths. These procedures start from the assumption that the weighting factors, but not the fluorescence lifetimes, change with the wavelength so that the parameters of the fluorescence decay are obtained from an over-determined (redundant) set of parametric surfaces. Thus, the numerical stability is increased and the accuracy of the results improved ${ }^{36,37}$. However, even if the global analysis is more accurate and, in some cases, even faster than the common least-square techniques, it implies measurements of the same sample at different wavelengths, which is leading to a slow dataacquisition and errors introduced by instrumental instability.

A faster but equally accurate evaluation procedure for samples, whose fluorescence decay behaviour is not known or is supposed to be best described by a continuum-exponential, e.g. the fluorescence of proteins containing various tryptophan segments, is the iterative approximation of the data with a stretched-exponential function ${ }^{53}$. However, this technique is inadequate for FLIM experiments on samples, which contain a limited number of relevant chromophores, because it does not give any information about the fluorescence lifetimes of the individual components but only about the mean fluorescence lifetime and about the degree of "non-exponentiality" of the fluorescence decay.

Fast evaluation techniques of multiexponential FLIM data are usually up to 100 times faster than iterative techniques and are based on the rather cumbersome multi-linearization of the fluorescence decay. Among the multi-linearization techniques used (not only) in fluorescence spectroscopy and microscopy, the transforms in a complex space, e.g. Fourier- and Laplace-transforms, are the most common. However, their low numerical stability especially during the back-transform and the fact that they lead to under-determined sets of equations makes them impracticable.

A very stabile, accurate, and fast alternative to the transforms in complex space is based on a noniterative linearization technique in real-space (time-space) initially used to discriminate between biand tri-exponential decays in a small time-interval ${ }^{50}$. The stability and speed of this technique and of its advancements has extensively been discussed elsewhere ${ }^{50,54}$. 


\section{Bioscientific applications}

The applications of FLIM in the biosciences are manifold and reach from monitoring cellular parameters $\left(\mathrm{Ca}^{2+} 55-57\right.$ and $\mathrm{Na}^{+}$concentrations ${ }^{58}, \mathrm{pH}^{59-64}, \mathrm{pO}_{2}$ and ROS concentrations ${ }^{65-67}, \mathrm{pCO}_{2}$ ${ }^{68}$ ) and the distances between proteins on the nm-scale by means of FRET ${ }^{5,35,42,46-48,68-81}$ to observing central cellular processes in real time, e.g. interactions between proteins in living cells 48,82-86, photophysics of complexes involved in plant photosynthesis ${ }^{87}$ or the redox metabolism 38,50,88-90. In the last years, FLIM gained on interest in the biomedicine, as well ${ }^{27,49,78,91-96}$. For instance, a FLIM device for the clinical diagnosis of malign skin tissue is commercially available (DermaInspect, JenLab, Jena, Germany) ${ }^{27}$. In the following we do not intend to review all these applications, more we focus our attention on some experiments based on time-gated FLIM where single-beam as well as multi-focal TPLSM were used.

\subsection{Acidic mantle of artificial skin constructs}

Research and development of novel drugs for transdermal administration require - among others the testing of percutaneous penetration of the drug molecules across human skin. Prior to in vivo experiments, in vitro studies with excised human skin - or as a less appropriate alternative, with excised animal skin - have to be performed. Since excised animal skin is just an insufficient substitute of human skin due to species variations in morphology and functionality, i.e. in drug permeability, excised human skin is preferred although its resources are rather limited. Therefore the development of human skin models, which have the same properties (morphology and functionality, i.e. the barrier function) as the genuine human skin, is of particular significance. Very promising skin models are the three-dimensional artificial skin constructs (ASC), which similarly to the genuine skin consist of an epidermis of differentiated keratinocytes and of a dermis.

In the following we will focus our attention on the barrier function of the skin, i.e. its selective permeability, with respect to the delivery of ionisable drugs. The barrier function of the skin is mainly determined by the upper lipophilic layers of the epidermis, i.e. stratum corneum. Moreover, the acidic mantel of the skin is responsible for low values of $\mathrm{pH}$ on the skin surface. Thereby, a $\mathrm{pH}$ gradient of few $\mathrm{pH}$ units exists between the outer stratum corneum layers and the viable part of the epidermis characterised by a physiological $\mathrm{pH}$. Both factors influence the permeation profile of the ionisable drugs through the skin. As far as the $\mathrm{pH}$ gradient is concerned, it has been shown that a modification of the $\mathrm{pH}$ of the epidermis results in a modification of the ionisation state of the drugs and, thus, in a modified permeation profile. Consequently, in order to demonstrate the reliability of the artificial skin constructs as models of the genuine human skin in permeability studies, it is necessary to compare their $\mathrm{pH}$ gradient with that of genuine skin. 
We employed time-gating FLIM combined with TPLSM in order to obtain the three-dimensional distribution of the $\mathrm{pH}$ in the epidermis of the ASC stained with BCECF (2',7'-bis-(2-carboxyethyl)5/6-carboxyfluorescein ${ }^{64}$. Thereby, the samples were neither photodamaged nor mechanically damaged. BCECF is particularly appropriate as a $\mathrm{pH}$ indicator for studies in vital tissue because its fluorescence lifetime dramatically changes at small $\mathrm{pH}$-deviations around the physiological $\mathrm{pH}$, i.e. 7.4 (Fig. 5).

Insert Fig. 5

In order to insure a high accuracy in the determination of the $\mathrm{pH}$, we took into consideration that the fluorescence lifetime of BCECF may also be influenced by other factors than the $\mathrm{pH}$ such as refractive index $n$, viscosity $\eta$ or ions concentration. While the influence of viscosity and ions concentration on the fluorescence lifetime of BCECF is negligible, the fluorescence lifetime is inversely proportional to the fluorescence lifetime $n^{2}$. Therefore, a correction of the fluorescence lifetime images of the BCECF-stained ASC for the refractive index $\mathrm{n}$ is required and, thus, it is essential to determine the $\mathrm{n}$ gradient in the heterogeneous artificial epidermis. In FLIM experiments on ASC stained with coumarin 314, we determined the local refractive index of the artificial epidermis with high 3D resolution (Fig. 6). This local refractive index was used to correct the fluorescence lifetime images of BCECF-stained samples before converting them into pH-images.

\section{Insert Fig. 6}

Differences between the $\mathrm{pH}$ of the cell interior (between 6.0 and 7.2) and of the intercellular matrix (between 5.8 and 6.6) are observed all through the artificial epidermis. The $\mathrm{pH}$ of the cell interior in $13.48 \mu \mathrm{m}$ depth amounts to $7.2 \pm 0.1$, while the $\mathrm{pH}$ of the intercellular matrix is $6.3 \pm 0.2$. Only at the surface, both the cells and the intercellular matrix are characterised by the same acidic $\mathrm{pH}$ of 5.9 \pm 0.1 . The dependence of the $\mathrm{pH}$ on the depth of the epidermal layer is schematically plotted in Fig. 7. The $\mathrm{pH}$ of the cell interior rapidly increases in stratum corneum (0 to $10 \mu \mathrm{m})$ from 6.0 to 7.2 and remains constant $(\mathrm{pH}=7.2)$ in the deeper layers beyond the junction between stratum corneum and granulosum (below $12 \mu \mathrm{m}$ ). An increasing tendency shows also the $\mathrm{pH}$ of the intercellular matrix. However, in this case the $\mathrm{pH}$ increases all through the epidermis (depth z between 0 and $30 \mu \mathrm{m}$ ) and does not exceed a value of $6.6 \pm 0.1$ (Fig. 7). Qualitatively, the profile of the $\mathrm{pH}$ averaged over a $\mathrm{pH}$-image is similar to the $\mathrm{pH}$-gradients in the cell interior and in the intercellular matrix, respectively. 
Insert Fig. 7

By comparing the $\mathrm{pH}$ distribution, the $\mathrm{n}$ distribution and the morphology of the artificial skin constructs with data obtained for the genuine human skin, we can conclude that the ASC are very reliable yet simplified skin models ${ }^{64}$.

\section{2. $N A D(P) H$ redox metabolism}

In order to probe the cellular redox metabolism by means of FLIM, we used as signal mediators the fluorescing reduced forms of the coenzymes nicotinamide adenosine dinucleotide: NADH and nicotinamide adenosine dinucleotide phosphate: NADPH. These coenzymes are ubiquitous cellular electron carriers for enzyme-catalysed redox processes in the ATP (adenosine triphosphate) production as well as in reductive biosyntheses of macromolecules. In order to perform accurate FLIM experiments, we isolated the signal of $\mathrm{NAD}(\mathrm{P}) \mathrm{H}$ from that of other endogenous chromophores by exciting the samples at $760 \mathrm{~nm}$ and observing the emission at $460 \pm 20 \mathrm{~nm}$. Under these conditions, more than $98 \%$ of the fluorescence within cells originates from NAD(P)H ${ }^{97}$. This is also confirmed by th fact that the cellular autofluorescence is low within the nuclei but high in small organelles within the cytosol (see Fig. 8).

\section{Insert Fig. 8}

NAD(P)H exists in two different states within the cell: either free, i.e. it does not participate to any cellular process, or enzyme-bound, i.e. it participates to vital redox processes catalysed by the enzymes, to which it is bound to. Both states of $\mathrm{NAD}(\mathrm{P}) \mathrm{H}$ (free and enzyme-bound) fluoresce and are characterised by specific fluorescence lifetimes. Thus, by using biexponential FLIM we are able to extract from the NAD(P)H signal of MIN6 cells two major fluorescence lifetimes in each pixel of the image: that of free NAD(P)H at approx. 450 ps and that of enzyme-bound NAD(P)H at approx. 3000 ps.

\section{Insert Fig. 9}

In order to perform a rapidly biexponential evaluation, which first enables dynamical tracking of changes in the cellular function, we implemented the Prony method in FLIM ${ }^{50}$.

$$
F(t)=a_{\text {free }} \cdot e^{-t / \tau_{\text {free }}}+a_{\text {bound }} \cdot e^{-t / \tau_{\text {bound }}}
$$

Besides the two fluorescence lifetime images of the free resp. enzyme-bound $\mathrm{NAD}(\mathrm{P}) \mathrm{H}$, a further result of the biexponential FLIM evaluation of the cellular NAD(P)H fluorescence is the ratioimage (Fig. 9) ${ }^{50}$. It represents the contribution of the enzyme-bound $\mathrm{NAD}(\mathrm{P}) \mathrm{H}$ to the total 
fluorescence signal, i.e. $a_{\text {bound }} \tau_{\text {bound }} /\left(a_{\text {free }} \cdot \tau_{\text {free }}+a_{\text {bound }} \tau_{\text {bound }}\right)$. Since the fluorescence signal of the free resp. bound $\mathrm{NAD}(\mathrm{P}) \mathrm{H}$ is proportional to their concentration, the ratio-image mirrors the redox activity of the cell and, thus, the cellular redox metabolism.

While the fluorescence lifetime distribution of free $\mathrm{NAD}(\mathrm{P}) \mathrm{H}$ within the cell well reproduces the response function of the detection device, the distribution of the enzyme-bound $\mathrm{NAD}(\mathrm{P}) \mathrm{H}$ is considerably broader (histograms in Fig. 9). Moreover, within a cell there are regions with different fluorescence lifetimes for bound $\mathrm{NAD}(\mathrm{P}) \mathrm{H}$. All these observations suggest that, based on the fluorescence lifetime of the NAD(P)H-enzyme-complex, it might be possible to specifically detect enzymes within the cell during their catalytic function only based on the fluorescence lifetime of the corresponding NAD(P)H-enzyme-complexes, as previously predicted by extracellular measurements. Since the coenzymes NADH and NADPH cannot indicate a specific cellular process, while the partner-enzymes enable such selectivity, the specific enzyme-detection directly lead to the ability of specifically and dynamically visualising vital processes with submicron resolution and without disturbing the sensitive cellular balance.

\section{Overview and perspectives}

Modern bioscientific research fields head to a non-invasive and fast investigation of vital phenomena within intact biological systems with high spatial resolution down to the molecular level. Thus, principal requirements of the biosciences on high-end technology, in our case on the fluorescence microscopy techniques, are apart of the high optical performance, imaging speed in real time, visualisation of deep layers within organs and tissue as well as maximum gain on information by monitoring as many relevant molecular parameters as possible. Considering the last item, we referred in this work to a time-resolved fluorescence microscopy technique of greatest interest for the biosciences, i.e. the fluorescence lifetime imaging (FLIM). FLIM is a calibrationfree technique, which monitors both the fluorescence intensity and the fluorescence lifetime in an image.

Currently employed imaging methods, which use only the integral emission of the sample and its spectral information to investigate biological samples, are shortly reviewed and compared in this work. Thereafter, we can conclude that the wide-field microscopy is appropriate for routine works, e.g. cell screening, whereas the confocal one-photon microscopy is especially adequate for highresolution investigations in systems containing few cells, in which fast fluctuations are not expected. The only established method, which has the potential of fast imaging in intact organs or 
even in the living organism, is the two-photon laser scanning microscopy (TPLSM). As far as the imaging speed is concerned, we showed that the multi-focal TPLSM is the only stabile fluorescence microscopic technique, which allows real-time intravital measurements at high spatial resolutions, i.e. on the nm scale.

A detailed presentation and comparison of common FLIM techniques, i.e. homodyne- and heterodyne frequency-domain (FD) FLIM, time-correlated single-photon counting (TCSPC) and time-gated FLIM, shows that the great variety of bioscientific questions cannot be optimally answered by only one FLIM technique. Each of the discussed techniques has its special advantages for a certain research area: FD FLIM allows routine low-cost investigations, TCSPC is particularly appropriate for studies around the single-molecule level whereas time-gating techniques based on intensified CCD detection can and have been combined with the (very fast) multi-focal TPLSM and, thus, show the potential for real-time intravital fluorescence lifetime imaging. Although both homodyne and heterodyne FD FLIM based on ICCD detection as well as TCSPC based on multianode PMT detection are theoretically compatible with multi-focal TPLSM, the technical implementation is in both cases very complicated and has not been realised yet. Two bioscientifically relevant applications of time-gating FLIM based on ICCD detection combined with single-beam and multi-beam TPLSM are presented: the investigation of the pH-gradient in artificial skin constructs and the monitoring of NAD(P)H redox metabolism in MIN6 cells.

The measurement of the fluorescence lifetime in an image is the most common time-resolved fluorescence imaging technique. Nevertheless, especially in the last decade techniques based on the measurement of the fluorescence anisotropy, i.e. the behaviour of the fluorescence polarisation induced by the rotational diffusion of chromophores, have attracted the attention for bioscientific topics ${ }^{98}$. Directly measuring the rotational diffusion of molecules provides artefact-free and accurate information about the physical environment of the chromophore molecules, i.e. viscosity and binding to other (non-fluorescing) molecules. Since setups for fluorescence anisotropy imaging (FAIM) are identical to FLIM setups except for the polarisation analysers, i.e. simple optics which can easily be incorporated into a commercial filter wheel, we expect that complementary FLIM/FAIM experiments will become standard procedures in the future.

Furthermore, the very advantageous use of TPLSM leads to another perspective of great relevance for the biosciences, i.e. the polarisation-sensitive two-colour-two-photon microscopy. The basic idea is that molecules with identical absorption and emission spectra and with identical fluorescence lifetimes in a similar environment can still be distinguished if they have different symmetries, i.e. 
different transition tensors for the two-photon excitation ${ }^{99}$. Such a technique makes use of the fact that two-photon absorption spectra, contrary to one-photon absorption spectra, are strongly depending on the relative polarisation of the exciting photons. Thus, we expect that ATP fluorescence can be distinguished from DNS or proteomic-tryptophan fluorescence although their UV-absorption spectra and fluorescence lifetimes are very similar.

\section{Acknowledgement}

We acknowledge the Bundesministerium für Bildung und Forschung for financial support under grant 0313412C (Bioprofile Hannover/Braunschweig/Göttingen) and our partners from the Joint Optical Metrology Centre, Braunschweig, for fruitful discussions. 


\section{FIGURE CAPTIONS}

Fig. 1: Experimental TPLSM setup compatible with both single-beam and multi-beam scanning.

Fig. 2: Blood flow in capillary vessels of a balb/c mouse. A) 2-dimensional imaging; B) 3dimensional imaging.

Fig. 3: Schematic Jablonski diagram for the special case of two-photon excitation.

Fig. 4: Principle of data acquisition in time-gating FLIM based on intensified CCD cameras as detectors.

Fig. 5: Fluorescence lifetime image and corresponding $\mathrm{pH}$ image of a layer in the stratum granulosum of artificial skin constructs stained with BCECF. The diagram shows the dependence of the fluorescence lifetime of aqueous BCECF-solutions on the $\mathrm{pH}$.

Fig. 6: Fluorescence lifetime image and corresponding refractive index (n) image of a layer in the stratum granulosum of artificial skin constructs. The diagram displays the behaviour of the averaged refractive index with the depth-position within the ASC.

Fig. 7: Fluorescence lifetime images of artificial skin constructs stained with BCECF indicate the 3dimensional $\mathrm{pH}$ gradient, i.e. epidermal barrier. The dependence of the $\mathrm{pH}$ on the depth position within the tissue is displayed in the diagram.

Fig. 8: Endogenous fluorescence of MIN6 cells caused by the fluorescing coenzymes NADH and NADPH.

Fig. 9: Biexponential result of endogenous NAD(P)H fluorescence lifetime imaging in MIN6 cells. a) fluorescence lifetime of free $\mathrm{NAD}(\mathrm{P}) \mathrm{H}$; b) fluorescence lifetime of enzyme-bound $\mathrm{NAD}(\mathrm{P}) \mathrm{H}$; c) contribution of the bound $\mathrm{NAD}(\mathrm{P}) \mathrm{H}$ to the total fluorescence signal; d) distributions of the fluorescence lifetimes of the free and bound $\mathrm{NAD}(\mathrm{P}) \mathrm{H}$, respectively. 


\section{Reference List}

1. Swedlow, J. R.; Platani, M. Cell Struct. Funct. 2002, 27: 335

2. Evans, J. A.; Nishioka, N. S. Curr. Opin. Gastroenterol. 2005, 21: 578

3. Saito, F.; Takahama, Y. Tanpakushitsu Kakusan Koso 2004, 49: 1592

4. Diaspro, A.; Chirico, G.; Collini, M. Q. Rev. Biophys. 2005, 38: 97

5. Chen, Y.; Mills, J. D.; Periasamy, A. Differentiation 2003, 71: 528

6. Lakowicz, J. R.; Szmacinski, H.; Nowaczyk, K.; Berndt, K. W.; Johnson, M. Analytical Biochemistry 1992, 202: 316

7. Szmacinski, H.; Nowaczyk, K.; Berndt, K.; Lakowicz, J. R. Faseb Journal 1992, 6: A35

8. Gratton, E.; Breusegem, S.; Sutin, J.; Ruan, Q.; Barry, N. J. Biomed. Opt. 2003, 8: 381

9. Becker, W.; Bergmann, A.; Hink, M. A.; Konig, K.; Benndorf, K.; Biskup, C. Microsc. Res. Tech. 2004, 63: 58

10. Becker, W.; Bergmann, A.; Haustein, E.; Petrasek, Z.; Schwille, P.; Biskup, C.; Kelbauskas, L.; Benndorf, K.; Klocker, N.; Anhut, T.; Riemann, I.; Konig, K. Microsc. Res. Tech. 2006, 69: 186

11. Elson, D.; Requejo-Isidro, J.; Munro, I.; Reavell, F.; Siegel, J.; Suhling, K.; Tadrous, P.; Benninger, R.; Lanigan, P.; McGinty, J.; Talbot, C.; Treanor, B.; Webb, S.; Sandison, A.; Wallace, A.; Davis, D.; Lever, J.; Neil, M.; Phillips, D.; Stamp, G.; French, P. Photochem. Photobiol. Sci. 2004, 3: 795

12. Gerritsen, H. C.; Vroom, J. M.; de Grauw, C. J. IEEE Eng Med. Biol. Mag. 1999, 18: 31

13. Behnsen, J.; Narang, P.; Hasenberg, M.; Gunzer, F.; Bilitewski, U.; Klippel, N.; Rohde, M.; Brock, M.; Brakhage, A. A.; Gunzer, M. PLoS. Pathog. 2007, 3: e13

14. Gunzer, M.; Riemann, H.; Basoglu, Y.; Hillmer, A.; Weishaupt, C.; Balkow, S.; Benninghoff, B.; Ernst, B.; Steinert, M.; Scholzen, T.; Sunderkotter, C.; Grabbe, S. Blood 2005, 106: 2424

15. Gunzer, M.; Weishaupt, C.; Hillmer, A.; Basoglu, Y.; Friedl, P.; Dittmar, K. E.; Kolanus, W.; Varga, G.; Grabbe, S. Blood 2004, 104: 2801

16. Denk, W.; Strickler, J. H.; Webb, W. W. Science 1990, 248: 73

17. Inoue, H.; Kudo, S. E.; Shiokawa, A. Nat. Clin. Pract. Gastroenterol. Hepatol. 2005, 2: 31

18. Miyashita, T. Methods Mol. Biol. 2004, 261: 399

19. Kaufman, S. C.; Musch, D. C.; Belin, M. W.; Cohen, E. J.; Meisler, D. M.; Reinhart, W. J.; Udell, I. J.; Van Meter, W. S. Ophthalmology 2004, 111: 396

20. Hell, S. W.; Andresen, V. J. Microsc. 2001, 202: 457

21. Nielsen, T.; Fricke, M.; Hellweg, D.; Andresen, P. J. Microsc. 2001, 201: 368 
22. Niesner, R. A.; Andresen, V.; Neumann, J.; Spiecker, H.; Gunzer, M. Biophys. J. 2007,

23. Egner, A.; Hell, S. W. Trends Cell Biol. 2005, 15: 207

24. Kano, H.; Jakobs, S.; Nagorni, M.; Hell, S. W. Ultramicroscopy 2001, 90: 207

25. Hell, S. W.; Schrader, M.; van, d., V , J. Microsc. 1997, 187: 1

26. Lakowicz, J. R. Principles of Fluorescence Spectrsocopy; 2. edition ed.; Kluwer Academic/Plenum Publishers: New York, Moskow, 1999.

27. Konig, K.; Riemann, I. Journal of Biomedical Optics 2003, 8: 432

28. Lakowicz, J. R.; Gryczynski, I. Arabian Journal for Science and Engineering 1992, 17: 261

29. Dyba, M.; Klar, T. A.; Jakobs, S.; Hell, S. W. Applied Physics Letters 2000, 77: 597

30. Squire, A.; Verveer, P. J.; Bastiaens, P. I. J. Microsc. 2000, 197: 136

31. Squire, J.; Mueller, M. Rev. Scientific Instruments 2001, 72: 2855

32. Herman, P.; Maliwal, B. P.; Lin, H. J.; Lakowicz, J. R. J. Microsc. 2001, 203: 176

33. Bastiaens, P. I.; Squire, A. Trends Cell Biol. 1999, 9: 48

34. Squire, A.; Bastiaens, P. I. J. Microsc. 1999, 193: 36

35. Squire, A.; Verveer, P. J.; Rocks, O.; Bastiaens, P. I. J. Struct. Biol. 2004, 147: 62

36. Verveer, P. J.; Squire, A.; Bastiaens, P. I. Biophys. J. 2000, 78: 2127

37. Verveer, P. J.; Squire, A.; Bastiaens, P. I. J. Microsc. 2001, 202: 451

38. Konig, K.; So, P. T.; Mantulin, W. W.; Tromberg, B. J.; Gratton, E. J. Microsc. 1996, 183: 197

39. Herman, P.; Maliwal, B. P.; Lakowicz, J. R. Anal. Biochem. 2002, 309: 19

40. Becker, W.; Bergmann, A.; Biskup, C. Microsc. Res. Tech. 2007, 70: 403

41. De, B. P.; Owen, D. M.; Manning, H. B.; Talbot, C. B.; Requejo-Isidro, J.; Dunsby, C.; McGinty, J.; Benninger, R. K.; Elson, D. S.; Munro, I.; John, L. M.; Anand, P.; Neil, M. A.; French, P. M. Microsc. Res. Tech. 2007, 70: 481

42. Schonle, A.; Glatz, M.; Hell, S. W. Applied Optics 2000, 39: 6306

43. Systma J.; Vroom J.M.; de Grauw C.J.; Gerritsen H.C. J. Microsc. 1998, 191: 39

44. van Geest L.K.; Boddeke F.R.; van Dijk P.W.; Kamp A.F; van der Oord C.J.R.; Stoop K.W.J. Proc. SPIE 1999,

45. Gerritsen, H. C.; Asselbergs, M. A.; Agronskaia, A. V.; Van Sark, W. G. J. Microsc. 2002, 206: 218 
46. Biskup, C.; Zimmer, T.; Kelbauskas, L.; Hoffmann, B.; Klocker, N.; Becker, W.; Bergmann, A.; Benndorf, K. Microsc. Res. Tech. 2007, 70: 442

47. Breusegem, S. Y.; Levi, M.; Barry, N. P. Nephron Exp. Nephrol. 2006, 103: e41

48. Biskup, C.; Kelbauskas, L.; Zimmer, T.; Benndorf, K.; Bergmann, A.; Becker, W.; Ruppersberg, J. P.; Stockklausner, C.; Klocker, N. J. Biomed. Opt. 2004, 9: 753

49. Dowling, K.; Dayel, M. J.; Lever, M. J.; French, P. M. W.; Hares, J. D.; Dymoke-Bradshaw, A. K. L. Optics Letters 1998, 23: 810

50. Niesner, R.; Peker, B.; Schlüsche, P.; Gericke, K. H. Chemphyschem. 2004, 5: 1141

51. Esposito, A.; Gerritsen, H. C.; Wouters, F. S. Biophys. J. 2005, 89: 4286

52. Pelet, S.; Previte, M. J.; Laiho, L. H.; So, P. T. Biophys. J. 2004, 87: 2807

53. Lee, K. C.; Siegel, J.; Webb, S. E.; Leveque-Fort, S.; Cole, M. J.; Jones, R.; Dowling, K.; Lever, M. J.; French, P. M. Biophys. J. 2001, 81: 1265

54. Zhang Z.Y.; Sun T.; Grattan K.T.V.; Palmer A.W. Proc. SPIE 1997, 2980: 90

55. Agronskaia, A. V.; Tertoolen, L.; Gerritsen, H. C. J. Biomed. Opt. 2004, 9: 1230

56. Lakowicz, J. R.; Szmacinski, H.; Nowaczyk, K.; Johnson, M. L. Cell Calcium 1992, 13: 131

57. Szmacinski, H.; Gryczynski, I.; Lakowicz, J. R. Photochem. Photobiol. 1993, 58: 341

58. Szmacinski, H.; Lakowicz, J. R. Anal. Biochem. 1997, 250: 131

59. Behne, M. J.; Meyer, J. W.; Hanson, K. M.; Barry, N. P.; Murata, S.; Crumrine, D.; Clegg, R. W.; Gratton, E.; Holleran, W. M.; Elias, P. M.; Mauro, T. M. J. Biol. Chem. 2002, 277: 47399

60. Behne, M. J.; Barry, N. P.; Hanson, K. M.; Aronchik, I.; Clegg, R. W.; Gratton, E.; Feingold, K.; Holleran, W. M.; Elias, P. M.; Mauro, T. M. J. Invest Dermatol. 2003, 120 : 998

61. Hanson, K. M.; Behne, M. J.; Barry, N. P.; Mauro, T. M.; Gratton, E.; Clegg, R. M. Biophys. J. 2002, 83: 1682

62. Lin, H. J.; Herman, P.; Kang, J. S.; Lakowicz, J. R. Anal. Biochem. 2001, 294: 118

63. Lin, H. J.; Herman, P.; Lakowicz, J. R. Cytometry A 2003, 52: 77

64. Niesner, R.; Peker, B.; Schlusche, P.; Gericke, K. H.; Hoffmann, C.; Hahne, D.; MüllerGoymann, C. Pharm. Res. 2005, 22: 1079

65. Stehning C.; Holst G. Proc. SPIE 2001,

66. Szmacinski, H.; Castellano, F. N.; Terpetschnig, E.; Dattelbaum, J. D.; Lakowicz, J. R.; Meyer, G. J. Biochim. Biophys. Acta 1998, 1383: 151

67. Murtaza, Z.; Herman, P.; Lakowicz, J. R. Biophys. Chem. 1999, 80: 143 
68. Herman, P.; Murtaza, Z.; Lakowicz, J. R. Anal. Biochem. 1999, 272: 87

69. Chen, Y.; Periasamy, A. Microsc. Res. Tech. 2004, 63: 72

70. Elangovan, M.; Day, R. N.; Periasamy, A. Biotechniques 2002, 32: 1260

71. Elangovan, M.; Day, R. N.; Periasamy, A. J. Microsc. 2002, 205: 3

72. Elangovan, M.; Wallrabe, H.; Chen, Y.; Day, R. N.; Barroso, M.; Periasamy, A. Methods 2003, 29: 58

73. Hoefelschweiger, B. K.; Pfeifer, L.; Wolfbeis, O. S. Journal of Biomolecular Screening 2005, 10: 687

74. Jose, M.; Nair, D. K.; Reissner, C.; Hartig, R.; Zuschratter, W. Biophys. J. 2007, 92: 2237

75. Murata, S.; Herman, P.; Lakowicz, J. R. J. Histochem. Cytochem. 2001, 49: 1443

76. Murata, S.; Herman, P.; Lakowicz, J. R. Cytometry 2001, 43: 94

77. Murata, S.; Herman, P.; Mochizuki, K.; Nakazawa, T.; Kondo, T.; Nakamura, N.; Lakowicz, J. R.; Katoh, R. J. Histochem. Cytochem. 2003, 51: 951

78. Murata, S.; Herman, P.; Iwashina, M.; Mochizuki, K.; Nakazawa, T.; Kondo, T.; Nakamura, N.; Lakowicz, J. R.; Katoh, R. J. Biomed. Opt. 2005, 10: 034008

79. Nair, D. K.; Jose, M.; Kuner, T.; Zuschratter, W.; Hartig, R. Optics Express 2006, 14: 12217

80. Pepperkok, R.; Squire, A.; Geley, S.; Bastiaens, P. I. Curr. Biol. 1999, 9: 269

81. Periasamy, A.; Elangovan, M.; Elliott, E.; Brautigan, D. L. Methods Mol. Biol. 2002, 183: 89

82. Esposito, A.; Dohm, C. P.; Kermer, P.; Bahr, M.; Wouters, F. S. Neurobiol. Dis. 2007, 26: 521

83. Jia, Y.; Sytnik, A.; Li, L.; Vladimirov, S.; Cooperman, B. S.; Hochstrasser, R. M. Proc. Natl. Acad. Sci. U. S. A 1997, 94: 7932

84. Schuttpelz, M.; Müller, C.; Neuweiler, H.; Sauer, M. Anal. Chem. 2006, 78: 663

85. Scully A.D.; Ostler R.B.; Phillips D.; O'Neill P.; Townsend K.; Parker A.W.; MacRobert A.J. Bioimaging 1997, 5: 9

86. Treanor, B.; Lanigan, P. M.; Suhling, K.; Schreiber, T.; Munro, I.; Neil, M. A.; Phillips, D.; Davis, D. M.; French, P. M. J. Microsc. 2005, 217: 36

87. Barzda, V.; de Grauw, C. J.; Vroom, J.; Kleima, F. J.; van, G. R.; van, A. H.; Gerritsen, H. C. Biophys. J. 2001, 81: 538

88. Evans, N. D.; Gnudi, L.; Rolinski, O. J.; Birch, D. J.; Pickup, J. C. Diabetes Technol. Ther. 2003, 5: 807 
89. Lakowicz, J. R.; Szmacinski, H.; Nowaczyk, K.; Johnson, M. L. Proceedings of the National Academy of Sciences of the United States of America 1992, 89: 1271

90. Schweitzer, D.; Schenke, S.; Hammer, M.; Schweitzer, F.; Jentsch, S.; Birckner, E.; Becker, W.; Bergmann, A. Microsc. Res. Tech. 2007, 70: 410

91. Esposito, A.; Gerritsen, H. C.; Oggier, T.; Lustenberger, F.; Wouters, F. S. J. Biomed. Opt. 2006, 11: 34016

92. Esposito, A.; Dohm, C. P.; Bahr, M.; Wouters, F. S. Mol. Cell Proteomics. 2007,

93. Evans, N. D.; Gnudi, L.; Rolinski, O. J.; Birch, D. J.; Pickup, J. C. J. Photochem. Photobiol. B 2005, 80: 122

94. Halbhuber, K. J.; König, K. Annals of Anatomy-Anatomischer Anzeiger 2003, 185: 1

95. Pfeifer, L.; Stein, K.; Fink, U.; Welker, A.; Wetzl, B.; Bastian, P.; Wolfbeis, O. S. Journal of Fluorescence 2005, 15: 423

96. Tadrous, P. J.; Siegel, J.; French, P. M.; Shousha, S.; Lalani, e.; Stamp, G. W. J. Pathol. 2003, 199: 309

97. Huang, S.; Heikal, A. A.; Webb, W. W. Biophys. J. 2002, 82: 2811

98. Benninger, R. K.; Onfelt, B.; Neil, M. A.; Davis, D. M.; French, P. M. Biophys. J. 2005, 88: 609

99. McClain W.M. Journal of Chemical Physics 1972, 57: 2264 